\title{
SUPERCONDUCTING PROPERTIES OF THE CHARGED WEAKLY INTERACTING BOSE GAS ("d" SYMMETRY)
}

\author{
Z.M. GaLASIEWICZ \\ Institute of Physics, Wrocław University of Technology \\ Wybrzeze Wyspiańskiego 27, 50-370 Wrocław, Poland \\ (Received February 2, 2000)
}

A system of charged weakly interacting bosons with " $d$ " symmetry is considered. The interaction $U^{(d)}=U^{(s)} f(\theta)$ contains the angle dependence $f(\theta) \sim\left|Y_{2,1}\right|^{2}$ consistent with " $d$ " symmetry. This system resembles much more free bosons than the system with " $s$ " symmetry (interaction $U^{(s)}$ ). For example at $T=0, n \approx n_{c}^{(d)}$, where $n, n_{c}^{(d)}$ are the densities of particles and condensate respectively.

PACS numbers: 67.40.-w, 74.20.-z

Most results of the recent experiments, specially for the cuprate high temperature superconductors, are consistent with the " $d$-wave" symmetry $[1,2]$. More precisely, important is the particular form of the " $d$-wave" known as the $d_{x^{2}-y^{2}}$ one, connected with $l=2$ and $m \pm 1$.

Superconducting properties of the charged free and weakly interacting Bose gas with " $s$ " symmetry were considered in Refs. [3-5] respectively. The ground state of the weakly interacting bosons has been considered already in 1947 by Bogolyubov [6].

For the case of " $s$ " symmetry the interaction was described by $U^{(s)}=2 \pi \hbar^{2} a / m$, where " $a$ " denotes the scattering length. In case considered here " $d$ " symmetry $U^{(d)}$ contains the angle dependence $f(\theta)$ proportional to $\frac{1}{2}\left(Y_{2,1} Y_{2,1}^{*}+Y_{2,-1} Y_{2,-1}^{*}\right)$, see e.g. [7], where $Y_{2, \pm 1} \sim \sin \theta \cos \theta \exp ( \pm \mathrm{i} \phi), l=2$, $m= \pm 1$ i.e.

$$
U^{(d)}=U^{(s)} f(\theta), \quad f(\theta)=(\sin \theta \cos \theta)^{2}, \quad \sqrt{f(\theta)}=|\sin \theta \cos \theta|>0 .
$$

The considered now system is described by the Hamiltonian

$$
\begin{aligned}
& \widehat{H}=E_{0}+\sum_{p \neq 0} \frac{p^{2}}{2 m} a_{p}^{+} a_{p}+U^{(s)} \frac{N}{V} \sum_{p \neq 0} f(\theta)\left(a_{p} a_{-p}+a_{-p}^{+} a_{p}^{+}+2 a_{p}^{+} a_{p}\right), \\
& U^{(s)} \frac{N}{V}=\frac{m u^{2}}{2}, \quad u=\frac{\sqrt{\pi a N / V} 2 \hbar}{m},
\end{aligned}
$$

where " $u$ " is the sound velocity. 
After Bogolyubov [6] unitary transformation $\left(a^{+}, a\right) \rightarrow\left(b^{+}, b\right)$ the diagonalized Hamiltonian (2) has the form

$$
\widehat{H}=\text { const }+\sum_{p \neq 0} \varepsilon(p, \theta) b_{p}^{+} b_{p}, \quad \varepsilon(p, \theta)=\sqrt{\left(\frac{p^{2}}{2 m}\right)^{2}+(u p)^{2} f(\theta)} .
$$

On the basis of the flow properties one can express the density of bosons $n$ as a sum of the density of the normal component $n_{\mathrm{n}}$ and the density $n_{\mathrm{s}}$ of the superfluid component $\left(n=n_{\mathrm{s}}+n_{\mathrm{n}}\right)$.

On the other hand, after use of the Bogolyubov transformation to the operator $a_{p}^{+} a_{p}$ one can, like in [4], introduce the alternative decomposition of the density $n$. Namely

$$
\begin{aligned}
n= & n_{\mathrm{n}}^{(d)}(\gamma, T)+n_{\mathrm{s}}^{(d)}(\gamma, T)=\frac{1}{V} \sum_{p} a_{p}^{+} a_{p} \\
& =n_{\mathrm{c}}^{(d)}(\gamma, T)+n_{\mathrm{int}}^{(d)}(u)+n_{\mathrm{ex}}^{(d)}(\gamma, T), \quad \gamma=\frac{m u^{2}}{k_{B} T} .
\end{aligned}
$$

(The dimensionless parameter $\gamma$ was denoted in $[4,5]$ by " $s$ ".)

In Eq. (4) $n_{\mathrm{c}}^{(d)}(\gamma, T)$ describes the density of the Bose condensate. The density $n_{\text {int }}^{(d)}$ presents bosons which due to interactions $U^{(d)}$ are at $T=0$ not in condensate. In case of noninteracting bosons at $T=0,100 \%$ of particles is in the Bose condensate. On the other hand, in superfluid $\mathrm{He}^{4}$ at $T=0$, because of strong interaction, $90 \%$ of particles is not in the condensate.

Finally, $n_{\mathrm{ex}}^{(d)}(\gamma, T)$ is the excitations density described by the Bose distribution function.

Now, in case of interaction $U^{(d)}(\theta)$ we have for $n_{\text {int }}^{(d)}$ the following expression:

$$
n_{\mathrm{int}}^{(d)}(u)=\left.\frac{1}{2 \pi^{2} \hbar^{3}} \int_{0}^{L} p^{2} \mathrm{~d} p \frac{1}{2} \int_{0}^{\pi}\left[\frac{p^{2}}{2 m}+m u^{2} f(\theta)-\varepsilon(p, \theta)\right] \frac{\sin \theta \mathrm{d} \theta}{2 \varepsilon(p, \theta)}\right|_{L \rightarrow \infty}
$$

$$
=n_{\text {int }}^{(s)}(u) \stackrel{(1)}{I^{(d)}}=0.057 n_{\text {int }}^{(s)}(u),
$$

$I^{(1)}=\frac{1}{2} \int_{0}^{\pi} f(\theta) \sqrt{f(\theta)} \sin \theta \mathrm{d} \theta=\frac{1}{2} \int_{0}^{\pi} \sin ^{4} \theta\left|\cos ^{3} \theta\right| \mathrm{d} \theta=0.057$.

We see that in case of " $d$ " symmetry $n_{\text {int }}^{(d)}$ is two orders of magnitude smaller than $n_{\text {int }}^{(s)}$.

For $n_{\mathrm{ex}}(\gamma, T)$ appearing in (4), using formula for critical temperature $T_{\mathrm{c}}^{0}$ for free Bose gas, we have

$$
\begin{aligned}
& n_{\mathrm{ex}}^{(d)}(\gamma, T)=\frac{\left(m k_{\mathrm{B}} T\right)^{3 / 2}}{2^{1 / 2} \pi^{2} \hbar^{3}} J^{(d)}(\gamma)=n\left(\frac{T}{T_{\mathrm{c}}^{0}}\right)^{3 / 2} \frac{J^{(d)}(\gamma)}{J(0)} \\
& J(0)=\int_{0}^{\infty} \frac{x^{1 / 2} \mathrm{~d} x}{\mathrm{e}^{x}-1}=2.315
\end{aligned}
$$


where

$$
\begin{aligned}
J^{(d)}(\gamma) & =\int_{0}^{\infty} \frac{x \mathrm{~d} x}{\mathrm{e}^{x}-1} \stackrel{(2)}{I}(x, \gamma) \\
& =\gamma^{3 / 2} \int_{0}^{\infty} \frac{z \mathrm{~d} z}{\left(\sqrt{z^{2}+1}+1\right)^{1 / 2}} \frac{1}{2} \int_{0}^{\pi} \frac{f(\theta) \sqrt{f(\theta)} \sin \theta \mathrm{d} \theta}{\mathrm{e}^{z \gamma f(\theta)}-1},
\end{aligned}
$$

and

$$
\begin{aligned}
& \stackrel{(2)}{I}(x, \gamma)=\frac{1}{2} \int_{0}^{\pi} \frac{\sin \theta \mathrm{d} \theta}{\left[\sqrt{x^{2}+\gamma^{2} f^{2}(\theta)}+\gamma f(\theta)\right]^{1 / 2}}=\frac{1}{\sqrt{x}}-\stackrel{(3)}{I}(x, \gamma), \\
& \stackrel{(3)}{I}(x, \gamma)=\frac{\gamma}{2} \int_{0}^{\pi} \frac{\cos ^{2} \theta\left(\cos ^{2} \theta-\sin ^{2} \theta\right) \sin \theta \mathrm{d} \theta}{\left[\sqrt{x^{2}+\gamma^{2} f^{2}(\theta)}+\gamma f(\theta)\right]^{3 / 2}} \\
& \times\left[\frac{\gamma f(\theta)}{\sqrt{x^{2}+\gamma^{2} f^{2}(\theta)}}+1\right]>0 .
\end{aligned}
$$

Finally, we have

$$
J^{(d)}(\gamma)=J(0)-\int_{0}^{\infty} \frac{x \mathrm{~d} x}{\mathrm{e}^{x}-1} \stackrel{(3)}{I}(x, \gamma)
$$

From (8), (9) and form of $I^{(s)}(\gamma)$ (see [4]) follows

$$
J(0)>J^{(d)}(\gamma)>J^{(s)}(\gamma)
$$

The second version of $J^{(d)}(\gamma)$, formula $(7)$, is convenient for a power series expansion in powers of $\gamma f(\theta)$.

Formula (6) shows that $n_{\mathrm{ex}}^{(d)}$ vanishes at $T=0$ i.e.

$$
n_{\mathrm{ex}}^{(d)}(\gamma, 0)=0 \text {. }
$$

From (4), (5) and (12) follows that at $T=0$

$$
n=n_{\mathrm{c}}^{(d)}(\gamma, 0)+n_{\text {int }}^{(d)}(u)=n_{\mathrm{c}}^{(d)}(\gamma, 0)+0.057 n_{\text {int }}^{(s)}(u) \approx n_{\mathrm{c}}^{(d)}(\gamma, 0),
$$

where $n_{\text {int }}^{(s)}<n$. Therefore, at least $94 \%$ of bosons is in the Bose condensate. In comparison to the system with " $s$ " symmetry the system with the " $d$ " one behaves much more similar to the free bosons system.

As we mentioned above, the density of the normal component $n_{\mathrm{n}}(\gamma, T)$ is calculated from the flow properties of the excitations with energies $\varepsilon=\varepsilon(p, \theta)+p v$. In the power series development of the averaged current we consider only term proportional to $v$, where $v$ denotes relative velocity between normal and superfluid component.

In order to find relation between $n_{\mathrm{ex}}^{(d)}$ and $n_{\mathrm{n}}^{(d)}$ we rewrite expression for $n_{\mathrm{ex}}^{(d)}$ in the form (see [4])

$$
n_{\mathrm{ex}}^{(d)}(\gamma, T)=\frac{\sqrt{2}(m u)^{3}}{3 \pi^{2} \hbar^{3}} J^{(d)}(\gamma), \quad(m u)^{3}=\left(m k_{\mathrm{B}} T\right)^{3 / 2} \gamma^{3 / 2}
$$

Here $I^{(d)}(\gamma)$ is given in terms of the second version presented in (7). 
For the density of the normal component we have

$$
\begin{aligned}
& n_{\mathrm{n}}^{(d)}(\gamma, T)=\frac{\sqrt{2}(m u)^{3}}{3 \pi^{2} \hbar^{3}} \frac{1}{2} \gamma^{3 / 2} \int_{0}^{\infty} \int_{0}^{\pi} \frac{f(\theta) \sqrt{f(\theta)} \sin \theta \mathrm{d} \theta \mathrm{d} z}{\mathrm{e}^{z \gamma f(\theta)}-1} \\
& \times \frac{\mathrm{d}}{\mathrm{d} z}\left[\frac{\left(\sqrt{z^{2}+1}-1\right)^{3 / 2} z}{\sqrt{z^{2}+1}}\right],
\end{aligned}
$$

where

$$
\begin{aligned}
\frac{\mathrm{d}}{\mathrm{d} z} & {\left[\frac{\left(\sqrt{z^{2}+1}-1\right)^{3 / 2} z}{\sqrt{z^{2}+1}}\right] } \\
& =\frac{3}{2} \frac{z}{\left(\sqrt{z^{2}+1}+1\right)^{1 / 2}}-\frac{\left(\sqrt{z^{2}+1}+2\right)\left(\sqrt{z^{2}+1}-1\right)^{1 / 2}}{2\left(\sqrt{z^{2}+1}\right)^{3}} .
\end{aligned}
$$

The first term of the right hand side of (16) appears in (7). From (7), (14), (15) and (16) follows

$$
n_{\mathrm{n}}^{(d)}(\gamma, T)=n_{\mathrm{ex}}^{(d)}(\gamma, T)-\frac{2}{3} n\left(\frac{T}{T_{\mathrm{c}}^{0}}\right)^{3 / 2} \gamma^{1 / 2} \frac{K^{(d)}(\gamma)}{J(0)} .
$$

The integral $K^{(d)}$ is expressed by the second term on the right hand side of (16) as follows:

Hence

$$
\begin{gathered}
K^{(d)}(\gamma)=\frac{\gamma}{2} \int_{0}^{\infty} \int_{0}^{\pi} \frac{f(\theta) \sqrt{f(\theta)} \sin \theta \mathrm{d} \theta \mathrm{d} z}{\mathrm{e}^{z \gamma f(\theta)}-1} \frac{\left(\sqrt{z^{2}+1}+2\right)\left(\sqrt{z^{2}+1}-1\right)^{1 / 2}}{2\left(\sqrt{z^{2}+1}\right)^{3}} \\
=\frac{\gamma^{3 / 2}}{2} \int_{0}^{\infty} \int_{0}^{\pi} \frac{\mathrm{d} z \sin \theta \mathrm{d} \theta}{\mathrm{e}^{z}-1} \\
\times \frac{\left[\sqrt{z^{2}+\gamma^{2} f^{2}(\theta)}+2 \gamma f(\theta)\right]\left[\sqrt{z^{2}+\gamma^{2} f^{2}(\theta)}-\gamma f(\theta)\right]^{1 / 2}}{2\left[\sqrt{z^{2}+\gamma^{2} f^{2}(\theta)}\right]^{3}}
\end{gathered}
$$

$$
n_{\mathrm{ex}}^{(d)}(\gamma, T) \geq n_{\mathrm{n}}^{(d)} .
$$

The first expression for $K^{(d)}$ in (18) is convenient for a power series expansion.

The critical temperature $T_{\mathrm{c}}$ is determined from the condition that the density of the condensate $n_{\mathrm{c}}^{(d)}(\gamma, T)$ should vanish at $T=T_{\mathrm{c}}$. From (4), (5) and (6) we have (see also (11))

$$
1=\frac{n_{\mathrm{int}}^{(d)}}{n}+\left(\frac{T_{\mathrm{c}}^{(d)}}{T_{\mathrm{c}}^{0}}\right)^{3 / 2} \frac{J^{(d)}\left(\gamma_{\mathrm{c}}, T_{\mathrm{c}}^{(d)}\right)}{J(0)}, \quad J(0)>J^{(d)} .
$$

Because $n_{\text {int }}^{(s)} / n<1$, it follows from (5) that $n_{\text {int }}^{(d)} / n=0.057 n_{\text {int }}^{(s)} \ll 1$ and can be neglected in (19). Equation (19) has now the form (see (11))

$$
\left(\frac{T_{\mathrm{c}}^{(d)}}{T_{\mathrm{c}}^{0}}\right)^{3 / 2}=\frac{J(0)}{J^{(d)}\left(\gamma_{\mathrm{c}}, T_{\mathrm{c}}^{(d)}\right)}>1, \quad T_{\mathrm{c}}^{(d)}>T_{\mathrm{c}}^{0} .
$$


For the " $s$ " symmetry considered in [4] there are estimations of the integrals $I^{(s)}(\gamma), K^{(s)}(\gamma)$ as a series expansion in powers of $\gamma$. Namely

$$
\begin{aligned}
& J^{(s)}(\gamma)=2.315-2.243 \gamma^{1 / 2}+1.294 \gamma-0.471 \gamma^{3 / 2}, \\
& K^{(s)}(\gamma)=1.122-0.706 \gamma
\end{aligned}
$$

After integration over angles of suitable terms in (21) we get approximate expressions for the case of " $d$ " symmetry. In case $J^{(s)}(\gamma) \rightarrow J^{(d)}(\gamma)$

$$
\gamma^{\alpha} \rightarrow \frac{1}{2} \gamma^{\alpha} \int_{0}^{\pi} f^{\alpha}(\theta) \sin \theta \mathrm{d} \theta
$$

and we have

$$
J^{(d)}(\gamma)=2.315-0.747 \gamma^{1 / 2}+0.172 \gamma-0.027 \gamma^{3 / 2}<J^{(s)}(\gamma) .
$$

In case $K^{(s)}(\gamma) \rightarrow K^{(d)}(\gamma)$

and

$$
\gamma^{\alpha} \rightarrow \frac{1}{2} \gamma^{\alpha} \int_{0}^{\pi} f^{\alpha+1 / 2}(\theta) \sin \theta \mathrm{d} \theta
$$

$$
K^{(d)}(\gamma)=0.374-0.05 \gamma<K^{(s)}(\gamma) .
$$

Now we will perform estimations of $(22)$, (23) for $\gamma$ in the interval $0 \leq \gamma \leq 0.5$. The mean values (arithmetic) $\bar{J}^{(s)}, \bar{J}^{(d)}$ for integrals $J^{(s)}(\gamma), J^{(d)}(\gamma)$ are

$$
\bar{J}^{(s)}=1.75, \quad \bar{J}^{(d)}=2.1 .
$$

This leads to the relations (see (20))

$$
T_{\mathrm{c}}^{(d)}=1.065 T_{\mathrm{c}}^{0} \approx T_{\mathrm{c}}^{0}, \quad \mathrm{~T}_{\mathrm{c}}^{(\mathrm{s})}=1.214 \mathrm{~T}_{\mathrm{c}}^{0} .
$$

The mean values of integrals $K^{(s)}(\gamma), K^{(d)}(\gamma)$ are

$$
\bar{K}^{(s)}=0.995, \quad \bar{K}^{(d)}=0.365=0.367 K^{(s)} .
$$

From (4), (5) we have

$$
n_{\mathrm{s}}^{(d)}-n_{\mathrm{c}}^{(d)}=n_{\mathrm{int}}^{(d)}+n_{\mathrm{ex}}^{(d)}-n_{\mathrm{n}}^{(d)}=0.057 n_{\mathrm{int}}^{(s)}+n_{\mathrm{ex}}^{(d)}-n_{\mathrm{n}}^{(d)} .
$$

From (16) and (27) follows

$$
n_{\mathrm{s}}^{(d)}-n_{\mathrm{c}}^{(d)}=0.057 n_{\mathrm{int}}^{(s)}+\frac{2}{3} n\left(\frac{T}{T_{\mathrm{c}}^{0}}\right)^{3 / 2} \gamma^{1 / 2} 0.367 \bar{K}^{(s)}<n_{\mathrm{s}}^{(s)}-n_{\mathrm{c}}^{(s)} .
$$

We see that there is more Bose condensate in the density of superfluid component $n_{\mathrm{s}}^{(d)}$ than in $n_{\mathrm{s}}^{(s)}$.

As concerns electrodynamics it was shown in [8] that in case of interaction $U^{(s)} \neq 0$ in the Schafroth [3] formula for supercurrent of free bosons the density of the condensate $n_{\mathrm{c}}$ should be replaced by the density of superfluid component $n_{\text {s. }}$. Namely $\left(U^{(s)} \neq 0\right)$

$$
j^{(s)}=-\frac{e^{2}}{m c} n_{\mathrm{s}}^{(s)} A \text {. }
$$

From (28) we see that especially when $T \rightarrow 0, j^{(d)}\left(U^{(d)} \neq 0\right)$ can be considered in good approximation as proportional to $n_{c}^{(d)}$ like in the Schafroth case for noninteracting bosons. 
From (13), (25), (28) follows that considered here system of weakly interacting bosons with " $d$ " symmetry $\left(U^{(d)}=U^{(s)} f(\theta)\right)$ resembles much more the system of free bosons than the system with " $s$ " symmetry $[4,5]$.

\section{References}

[1] D.J. Van Harlingen, Rev. Mod. Phys. 67, 515 (1995).

[2] B. Goss Levi, Phys. Today 49, 19 (1996).

[3] M.R. Schafroth, Phys. Rev. 100, 463 (1955).

[4] C.P. Enz, Z.M. Galasiewicz, Physica C 214, 239 (1993).

[5] Z.M. Galasiewicz, M. Wolf, Physica C 248, 49 (1995).

[6] N.N. Bogolyubov, J. Phys. (USSR) 11, 23 (1947).

[7] D.J. Blokhintzev, Foundations of Quantum Mechanics, Nauka, Moscow 1961, Ch. VIII (in Russian).

[8] Z.M. Galasiewicz, in: Recent Developments in High Temperature Superconductivity, Ed. J. Klamut, Springer-Verlag, Berlin 1996, p. 279. 